\title{
Efecto de Jatropha urens (Euphorbiaceae) y Lantana camara (Verbenaceae) sobre Trialeurodes vaporariorum (Hemiptera: Aleyrodidae)
}

\author{
EFFECT of Jatrophaurens (Euphorbiaceae) and Lantana camara (Verbenaceae) \\ on Trialeurodes vaporariorum (Hemiptera: Aleyrodidae)
}

\section{Natalia Vinasco A. ${ }^{1}$; Estefany Salazar . $^{2}$; Alberto Soto G. ${ }^{3}$; Luis Fernando Mejía G. ${ }^{4}$; Carmen Dussan L. ${ }^{5}$}

1 I.A. Universidad de Caldas. Manizales, Caldas, Colombia; vinasco.natalia @gmail.com

2 .A. Universidad de Caldas. Manizales, Caldas, Colombia; estefany.salazarpineda@gmail.com.

3 I.A. Ph.D. Profesor Universidad de Caldas. Manizales, Caldas, Colombia; alberto.soto@ucaldas.edu.co

4 I.Q., M.Sc. Profesor Universidad de Caldas. Manizales, Caldas, Colombia; lufermegu@gmail.com.

5 I.Q., M.Sc. Profesora Universidad de Caldas. Manizales, Caldas, Colombia; carmen.dussan@ucaldas.edu.co

Fecha recepción: Marzo 19 de 2015

Fecha de aceptación: Mayo 15 de 2015

\section{RESUMEN}

Trialeurodes vaporariorum es una de las principales plagas asociadas a cultivos hortícolas y su control se basa generalmente en el uso de agroquímicos. El objetivo de este trabajo, fue evaluar el efecto de los extractos de Jatropha urens y Lantana cámara y el producto comercial Alisin ${ }^{\circledast}$ sobre los estados de desarrollo de T. vaporariorum en condiciones de laboratorio. En huevos, el extracto etanólico de L. camara y acetónico de J. urens ocasionaron mortalidad superior al 33\%; en ninfas, los extractos etanólicos de ambas especies ocasionaron mortalidad superior al 94\%, mientras que en adultos, los extractos acetónicos de J. urens y acetónicos-etéricos de L. camara ocasionaron mortalidad superior al 80\%. El producto comercial Alisin ${ }^{\circledR}$ tuvo un comportamiento similar a los extractos acetónicos de J. urens y acetónicos-etéricos de L. cámara para el control de adultos de la plaga. El manejo de T. vaporariorum fue los extractos vegetales a base de J. urens y L. camara.

Palabras clave: Mosca blanca, Manejo integrado de plagas, extractos vegetales. 


\section{ABSTRACT}

Trialeurodes vaporariorum is one of the main pests associated to horticulture crops, and its control is generally based on the use of agrochemicals. The objective of this study was to evaluate the effect of $J$. urens and Lantana camara extracts and Alisin ${ }^{\circledR}$ commercial product on the developmental stages of T. vaporariorum under laboratory conditions. In eggs, the ethanolic extract of L. camara and the acetonic extract of J. Urens caused mortality above $33 \%$; in nymphs, the ethanolic extracts of both species caused mortality greater than $94 \%$, while in adults, the acetonic extracts of J. urens and the acetonic+ether extracts of L. camara caused mortality over $80 \%$. Alisin $\AA$ showed a similar behavior to the acetonic extracts of $J$. urens and the acetonic+ether extracts of L. camara for the control of pest adults.

Keywords: Whitefly, Integrated pest management, plant extracts.

\section{INTRODUCCIÓN}

La mosca blanca de los invernaderos T. vaporariorum Westwood (Hemiptera: Aleyrodidae), es una especie cosmopolita y polífaga (Van Lenteren y Noldus, 1990 y Hamdi et al., 2011) que ocasiona daños económicos a los cultivos al alimentarse de la savia, afectando la calidad de la planta y contribuyendo a la formación de fumagina y la transmisión de enfermedades virales (Byrne y Bellows, 1991; Van Lenteren et al., 1996; Park et al., 1998; Bi et al., 2002; Cardona et al., 2005; Arnó et al., 2006 y Hamdi et al., 2011).

Para el control de esta plaga los productores utilizan insecticidas sintéticos en grandes volúmenes sin cumplir los periodos de carencia, ocasionando residuos tóxicos en los frutos, desarrollo de poblaciones resistentes a los productos, destrucción de organismos benéficos, intoxicación de mamíferos y contaminación del medio ambiente (Fragoso et al., 2002; Isman, 2006; Picanço et al., 2007).
Feget (1994) menciona que con el fin de encontrar nuevas alternativas de manejo de plagas que busquen racionalizar el uso de agroquímicos, disminuir los costos de producción y obtener productos sanos, el uso de plantas con potencial insecticida es una herramienta valedera y eficiente para disminuir problemas de plagas en diferentes cultivos.

Una alternativa viable a los problemas ocasionados por el uso excesivo de insecticidas de síntesis química es la utilización de métodos de control que deben priorizar la seguridad ambiental y social y que sean eficientes en el control de T. vaporariorum. En la búsqueda de tales métodos, productos alternativos a los plaguicidas convencionales como los extractos vegetales, han sido utilizado por productores de hortalizas para el control de plagas y enfermedades (Van Lenteren et al., 1996; Simmonds et al., 2002; Choi et al., 2003; Fraser, 2005; Regnault-Roger et al., 2005; Pavela y Herda, 2007; Schuster et al., 2009; Hamdi et al., 2011; Moreau e Isman, 2012). 
Se han realizado pocos estudios con J. urens (Euphorbiaceae) para determinar su efecto insecticida. Dillon et al. (1983) observaron que las larvas de Erinnyis ello (Lepidoptera: Sphingidae) presentaron repelencia al alimentarse con hojas tratadas con dicha planta. Pérez (2012) estudió el efecto de Lantana camara (Verbenaceae) sobre Myzus persicae (Hemiptera: Aphididae) y Plutella xylostella (Lepidoptera: Plutellidae), observando que esta planta presenta actividad insecticida, debido a la presencia de triterpenos pentacíclicos y además compuestos sesquiterpenos (Zandi et al., 2012).

Lannacone y Lamas (2003) encontraron que los extractos de flores de L. camara presentaron repelencia contra Aedes aegypti (Diptera: Culicidae). El producto comercial Alisin $₫$, compuesto por los ingredientes activos de aji 10\% y ajo 10\% $(70 \mathrm{~g} / 1)$, se ha utilizado para el control de moscas blancas en diversos cultivos. Este posee compuestos como bisulfuro de alilo, limoneno, capsaicina, ácido nicotínico y carotenoides, los cuales presentan efecto de repelencia, disuasión de la alimentación y oviposición, interferencia con el crecimiento y desarrollo de los insectos plaga (Safer, 2014).

En este trabajo se evaluó el efecto insecticida que presenta J. urens y L. camara sobre huevos, ninfas y adultos de T. vaporariorum a partir de la extracción con los disolventes etanol, éter, acetona y acetona-éter en concentraciones del $100,75,50$ y $25 \%$, comparado con el producto comercial Alisin ${ }^{\circledR}$.

\section{MATERIALES Y MÉTODOS}

El trabajo se realizó en los laboratorios de Entomología y de Procesos Agroindustriales de la
Universidad de Caldas. La mosca blanca T. vaporariorum fue criada en casa malla, en plantas de fríjol variedad Calima de 30 días de edad, sembradas en bolsas de polietileno de $8 \times 12 \mathrm{~cm}$, acondicionas en jaulas de $80 \times 70 \times 50 \mathrm{~cm}$ de madera forradas con tela de muselina. La cría se inició con moscas colectadas en campo de la Granja Tesorito de la Universidad de Caldas.

Para la obtención de los extractos se utilizaron tallos y hojas de J. urens y de L. cámara, para lo cual se realizó un proceso de congelación lenta y posterior maceración pasiva durante $24 \mathrm{~h}$. La extracción se llevó a cabo por el método Soxhlet con los disolventes acetona, éter, etanol y acetona-éter, para luego ser llevada a un proceso de rotaevaporación al vacío en un rotovapor Buchi Water bath 114 a $40{ }^{\circ} \mathrm{C}$ de temperatura, con el fin de separar los disolventes de las sustancias extraídas. Los extractos obtenidos se empacaron en recipientes color ámbar y se almacenaron a una temperatura de $6{ }^{\circ} \mathrm{C}$.

Se evaluó el efecto de cada uno de los extractos sobre los estados de huevo, ninfas y adultos de T. vaporariorum a concentraciones de 100, 75, 50 y $25 \%$, con cinco repeticiones por tratamiento; para huevos y ninfas, la unidad experimental estuvo constituida por 50 individuos. Se utilizó el método de inmersión, el cual consistió en sumergir las hojas de frijol infestadas durante 1 min con cada uno de los tratamientos.

Posteriormente, se dejaron secar las hojas y se ubicaron en cámara BOD a $24^{\circ} \mathrm{C}$. Las evaluaciones se realizaron a las 24,48 y $72 \mathrm{~h}$, observando con la ayuda de un estereoscopio, la mortalidad ocasionada por cada uno de los tratamientos. 
Para los adultos, la unidad experimental para los ensayos estuvo conformada por 10 hembras adultas de T. vaporariorum colocadas sobre hojas de la planta de fríjol, en la cual se acondicionó una jaula pinza construida con dos tubos de PVC transparentes (diámetro = $2,5 \mathrm{~cm}$; profundidad $=2,0 \mathrm{~cm}$ ) a las cuales se les retiró la parte superior e inferior y se cubrieron con tela muselina. A la parte inferior del tubo se le realizó un orificio, donde se introdujeron los adultos de mosca blanca con la ayuda de un aspirador bucal, posteriormente se tapó dicho orificio con una mota de algodón. Los discos con las hojas tratadas fueron mantenidos en cámara climatizada a $25 \pm 2^{\circ} \mathrm{C}, 60 \pm 10 \% \mathrm{HR}$ y $13 \mathrm{~h}$ de luz. La mortalidad fue evaluada $24 \mathrm{~h}$ después de la aplicación de los productos.

Para cada estado de desarrollo de T. vaporariorum (huevos-ninfas-adultos) se analizó, mediante el Diseño Completamente Aleatorizado a una vía, comparaciones del porcentaje de mortalidad medio entre extractos. Se eligieron los dos extractos que presentaron mayor mortalidad para ambas plantas y que fueran significativamente diferentes de los demás. Además, se compararon los porcentajes de mortalidad medios de los extractos elegidos para cada estado de desarrollo de T. vaporariorum, contra el producto comercial Alisin ${ }^{\circledR}$. En cada etapa del análisis se validaron los supuestos de normalidad y homocedasticidad de los residuales, mediante las pruebas de Shapiro-Wilks y de Bartlett. En caso que alguno de los supuestos anteriores no fuera válido (o ambos), se aplicó la prueba no paramétrica de Kruskal-Wallis (Walpole et al., 2012). Las comparaciones entre tratamientos se realizaron utilizando la prueba LSD de Fisher (Sheskin, 2007). El programa estadístico utilizado fue Xlstat Versión 2014.

\section{RESULTADOS Y DISCUSIÓN}

Productos de menor riesgo como los extractos de plantas representan controles alternativos de plagas que son considerados de menor impacto para el ambiente y la salud que los pesticidas convencionales (Fraser, 2005). El presente estudio demostró que los extractos de las plantas $J$. urens y L. camara son prometedores para el control de $T$. vaporariorum debido a la mortalidad que se registró en los estados de ninfas y adultos. Además, se observó una reducción de la oviposición en los tratamientos, siendo éste un método recomendado para reducir las densidades de artrópodos plaga y daños a los cultivos (Cook et al., 2007; Foster y Harris, 1997).

Roel et al. (2000) afirman que la efectividad de un extracto botánico depende de la sustancia utilizada para su extracción (acetona ó etanol). Esto fue corroborado en el estado de huevos con la selección de los extractos J. urens + Acetona $50 \%$ y L. cámara + Etanol 100\%, los cuales presentaron mortalidad estadísticamente superior a los demás tratamientos. La actividad ovicida ocasionada por el extracto etanólico a una concentración del 100\% (E100) de L. camara sobre T. vaporariorum fue del 28\%, similar a lo observado por Aarthi y Murugan (2011), quienes a partir del extracto de Vetiveria zizanioides (Gramineae) obtenido con el disolventeetanol,encontró una respuesta positiva sobre la mortalidad de la mosca blanca. Para J. urens el extracto de acetona a una concentración 
del 50\% (A50) ocasionó mortalidad del 26\% (Figura 1).

Para el estado de ninfas de T. vaporariorum, se observó que el extracto de ambas plantas el etanólico a una concentración del 25\% logró mortalidad superior al 90\% (Figura 2), siendo superior estadísticamente a los demás tratamientos, similar a lo reportado por Rodríguez (2002) quien demostró que el Nim (Azadirachta indica) en el cultivo de algodón, ocasiona del 73 al 100\% de mortalidad en ninfas de la mosca blanca. Bouda et al. (2001) mostraron que los aceites esenciales de L. camara presentaron una tasa de mortalidad para Sitophilus zeamais (Coleoptera: Curculionidae) del 100\% a una concentración del 0,5\% en $48 \mathrm{~h}$.
El efecto insecticida sobre adultos de $T$. vaporariorum registró una mortalidad superior al $80 \%$ tanto para J. urens con el extracto acetónico al $50 \%$ y para L. camara con el extracto acetónicoéterico al 25\% (Figura 3), significativamente superiores a los demás extractos, valor diferente a lo obtenido por Gómez y Soto (2008) con la pringamosa (J. sp.) lográndose la mortalidad en el primer día con concentraciones superiores al $50 \%$, diferente a la concentración del $25 \%$ donde sólo en el día ocho ocasionó la muerte al 30\% de la población. Dehghani et al. (2012) registraron una disminución del 92,2 y 90,3\% de los adultos de T. vaporariorum con las plantas Melia azedarach (Meliaceae) y Peganum harmala (Nitrariaceae), respectivamente.

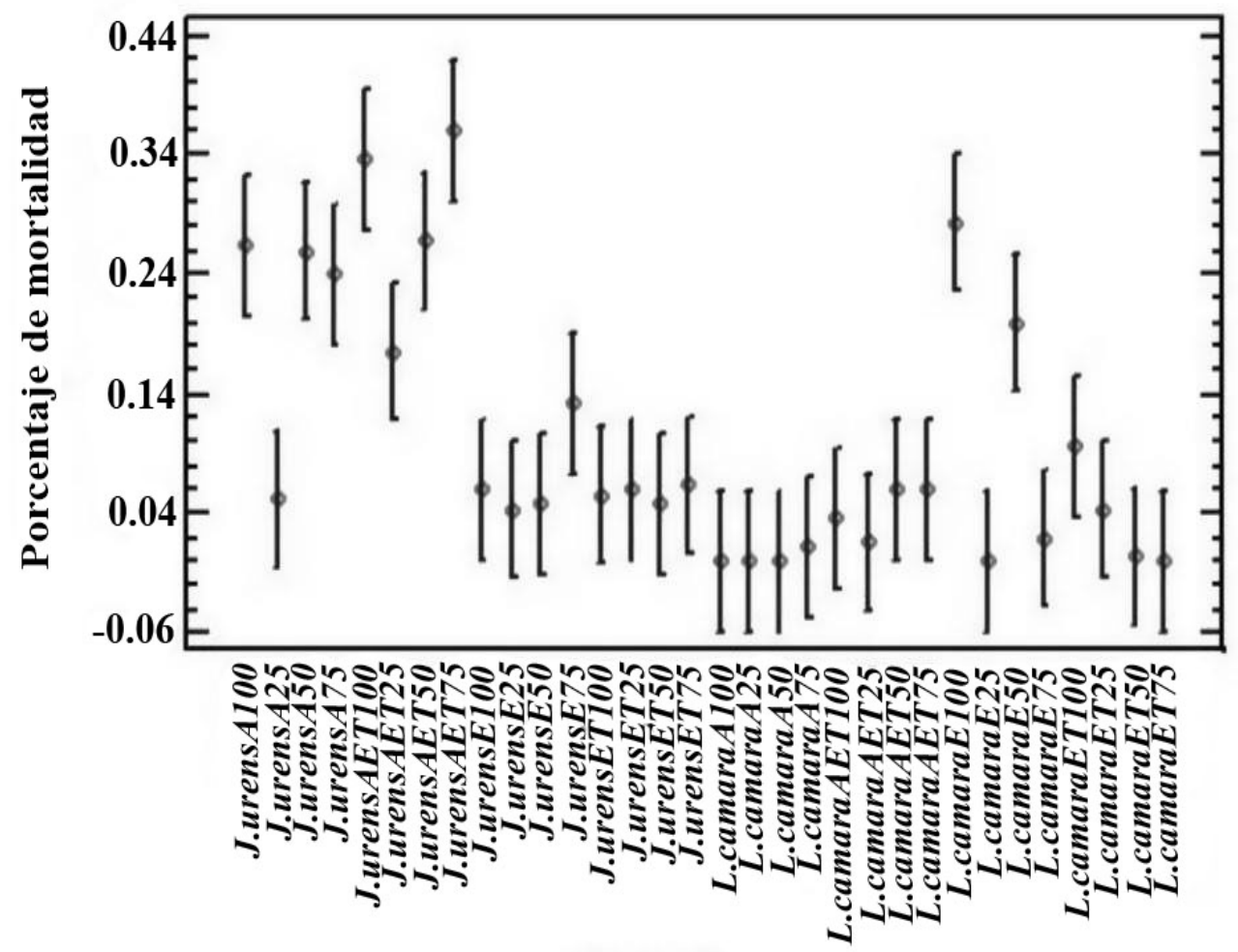

*A: acetona; AET: acetona + éter; E: etanol; ET: éter. * [ ]: 25, 50, 75,100

Figura 1. Mortalidad ocasionada por J. urens y L. cámara en diferentes concentraciones sobre huevos de T. vaporariorum. 


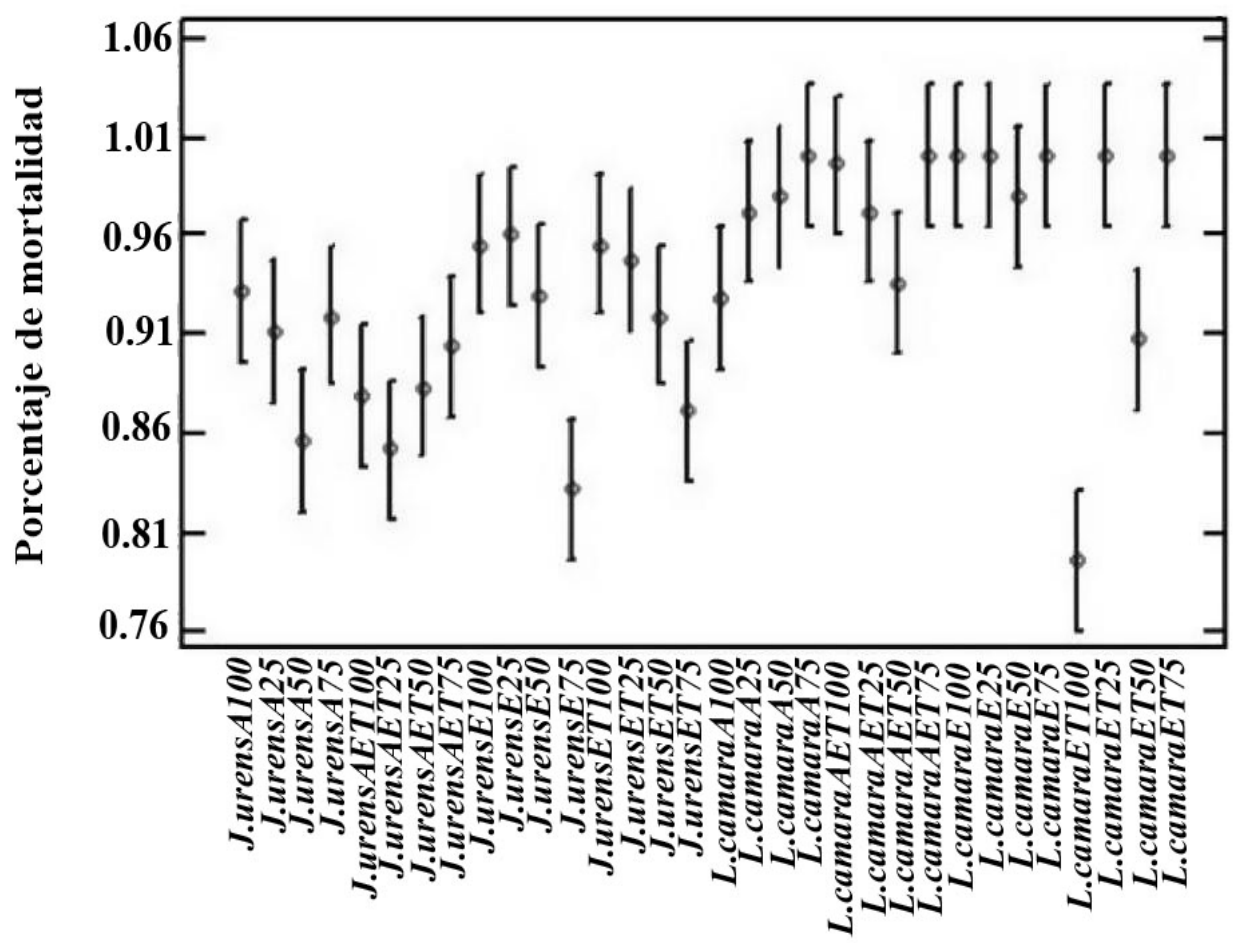

${ }^{*} \mathrm{~A}=$ acetona; $\mathrm{AET}=$ acetona + éter; $\mathrm{E}=$ etanol; $\mathrm{ET}=$ éter. ${ }^{*}[\mathrm{]}=25,50,75,100$

Figura 2. Mortalidad ocasionada por J. urens y L. cámara en diferentes concentraciones sobre ninfas de T. vaporariorum.

Para evaluar el comportamiento de J. urens y L. camara contra el producto comercial Alisin ${ }^{\circledR}$ se compararon los mejores tratamientos de los extractos para cada estado de desarrollo contra la concentración comercial de $2 \mathrm{cc} / 1$ de dicho producto. En el estado de huevo, el Alisin ${ }^{\circledR}$ produjo una mortalidad significativamente inferior a la reportada para J. urens con el tratamiento
Acetona $50 \%$ y a L. camara con el tratamiento Etanol 100\% (Tabla 1). Asi mismo, es claro que para éstos dos últimos tratamientos, la mortalidad media es estadísticamente igual. Salazar et al. (2003) indican que a las $72 \mathrm{~h}$, los extractos de ajo y ají ocasionan mortalidad del $98 \%$ y $87 \%$ sobre T. vaporariorum, siendo similar a los resultados obtenidos.

Tabla 1. Intervalos de confianza al $95 \%$ para la media de los tratamientos en huevos de T. vaporariorum.

\begin{tabular}{lcc}
\hline \multicolumn{1}{c}{ Tratamiento } & Límite Inferior & Límite Superior \\
\hline Alisin ${ }^{\circledR}$ & 0,033 & 0,135 \\
J. urens + Acetona $50 \%$ & 0,21 & 0,311 \\
L. camara + Etanol $100 \%$ & 0,23 & 0,335 \\
\hline
\end{tabular}




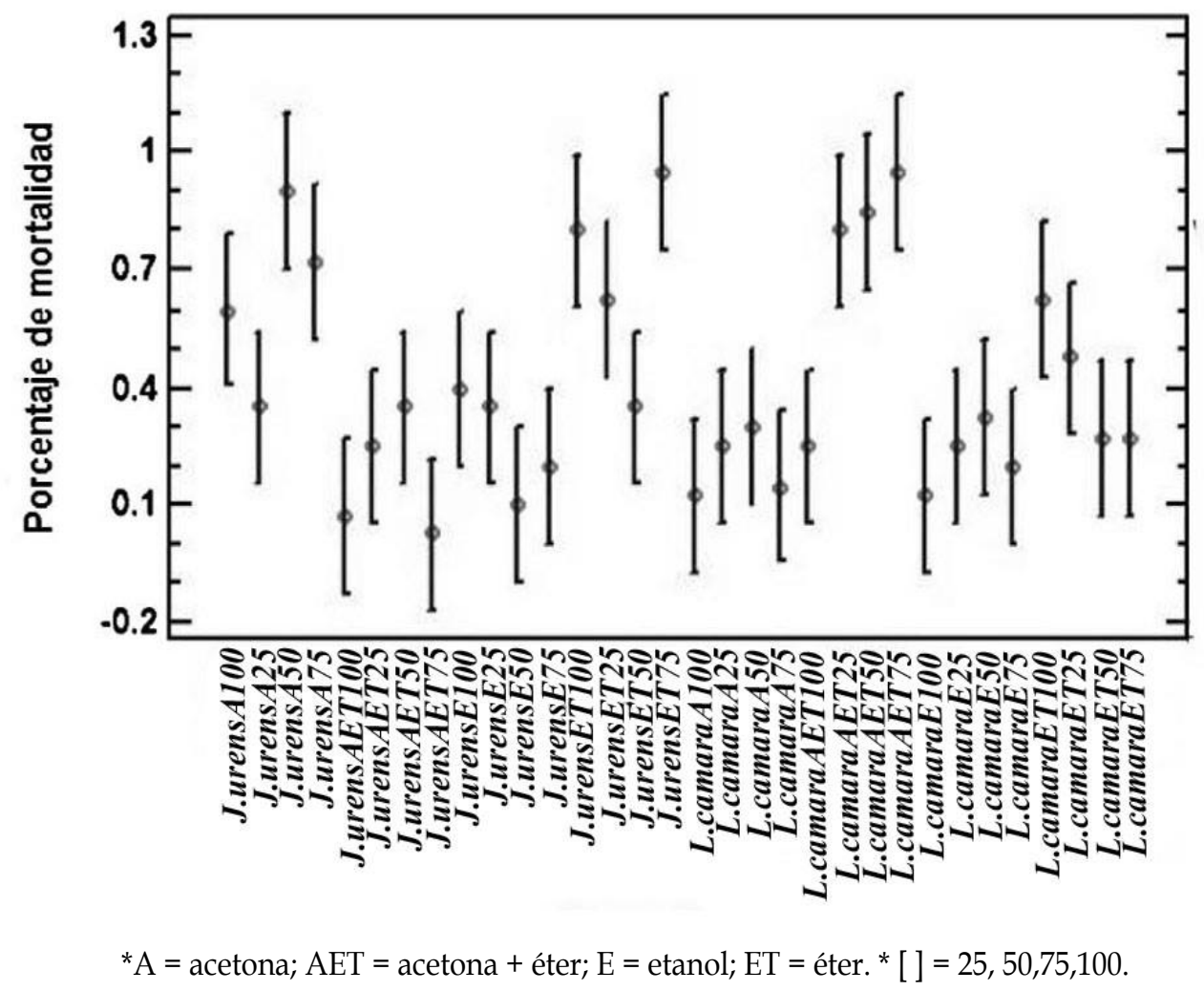

Figura 3. Mortalidad ocasionada por J. urens y L. cámara en diferentes concentraciones sobre adultos de T. vaporariorum.

Para evaluar el comportamiento de J. urens y $L$. camara contra el producto comercial Alisin ${ }^{\circledR}$ se compararon los mejores tratamientos de los extractos para cada estado de desarrollo contra la concentración comercial de $2 \mathrm{cc} / 1$ de dicho producto. En el estado de huevo, el Alisin ${ }^{\circledR}$ produjo una mortalidad significativamente inferior a la reportada para J. urens con el tratamiento Acetona $50 \%$ y a L. camara con el tratamiento Etanol 100\% (Tabla 1). Asi mismo, es claro que para éstos dos últimos tratamientos, la mortalidad media es estadísticamente igual. Salazar et al. (2003) indican que a las $72 \mathrm{~h}$, los extractos de ajo y ají ocasionan mortalidad del $98 \%$ y $87 \%$ sobre T. vaporariorum, siendo similar a los resultados obtenidos.

En el estado de ninfas, la Prueba de Fisher LSD indica que el tratamientos Alisin ${ }^{\circledR}$ contra el tratamiento J. urens + Etanol 25\% producen mortalidad estadísticamente iguales, e inferiores a la que produce el tratamiento L. camara + Etanol $25 \%$ (Tabla 2).

Para los adultos de T. vaporariorum se realizó la Prueba de Kruskal - Wallis, la cual muestra que no existe diferencia estadística entre los porcentajes de mortalidad medios entre los tratamientos.

L. cámara y J. urens representan una alternativa para el control de T. vaporariorum, especialmente cuando los agroquímicos no son permitidos, como en cultivos orgánicos. Sin embargo, la eficiencia de los productos alternativos para el control de plagas, así como la selectividad a enemigos naturales, está relacionada con la dosis 
Tabla 2. Intervalos de confianza al $95 \%$ para la media de los tratamientos en ninfas de T. vaporariorum.

\begin{tabular}{lcc}
\hline \multicolumn{1}{c}{ Tratamiento } & Límite Inferior & Límite Superior \\
\hline Alisin ${ }^{\circledR}$ & 0,912 & 0,944 \\
J. urens + Etanol $25 \%$ & 0,943 & 0,976 \\
L. camara + Etanol $25 \%$ & 0,983 & 1,000 \\
\hline
\end{tabular}

y la formulación empleada. Es necesario tener un conocimiento técnico sobre el producto que se va a utilizar para que se obtenga un control satisfactorio de las poblaciones plaga, de manera que no afecte a los enemigos naturales asociados a estas (Soto et al., 2011).

\section{CONCLUSIONES}

El efecto de J. urens y L. cámara sobre huevos y ninfas de T. vaporariorum registro una mortalidad mayor al $80 \%$, siendo estos extractos vegetales una alternativa de manejo importante para cultivos donde se presente reiteradamente la plaga. También, es interesante observar la capacidad de extracción de los disolventes utilizados (etanol, éter, acetona, éter-acetona) para obtener los metabolitos secundarios que causan la toxicidad en el insecto. Los extractos etanólicos y acetónicos fueron los más efectivos para obtener la $\mathrm{DL}_{50}$ en las poblaciones del insecto plaga a diferentes niveles de concentración.

\section{AGRADECIMIENTOS}

A la Vicerrectoría de Investigaciones de la Universidad de Caldas por la financiación de la investigación y a la empresa SLOANE Colombia por el suministro del material vegetal.

\section{REFERENCIAS}

AARTHI, N. y MURUGAN, K. 2011. Effect of Vetiveria zizanioides L. Root extracts on the malarial vector, Anopheles stephensi Liston. Asian Pacific Journal of Tropical Disease. 1(1):154 - 158.

ARNÓ, J., ALBAJES, R. y GABARRA, R. 2006. Within-plant distribution and sampling of single and mixed infestations of Bemisia tabaci and Trialeurodes vaporariorum (Homoptera: Aleyrodidae) in winter tomato crops. Journal Economic Entomology. 99(2):331 - 340 .

BI, J.L., TOSCANO, N.C. y BALLMER, G.R. 2002. Greenhouse and field evaluation of six novel insecticides against the greenhouse whitefly Trialeurodes vaporariorum on strawberries. Crop Protection. 21(1):49 - 55.

BOUDA. H., TAPONDJOU.L.A., FONTEM. D.A. y GUMEDZOE. M.Y. 2001. Effect of essential oils from leaves of Ageratum conyzoides, Lantana camara and Chromolaena odorata on the mortality of Sitophilus zeamais (Coleoptera, Curculionidae). Journal of Stored Products Research. 37(1):103 - 109.

BYRNE, D.N. y BELLOWS, T.S. 1991. Whitefly biology. Annual Review Entomology. 36:431 457.

CARDONA, C., RODRIGUEZ, V., BUENO, J. y TAPIA, X. 2005. Biología de la Mosca Blanca (Trialeurodes vaporariorum) en Habichuela y Frijol. Ed. Centro Internacional de Agricultura Tropical (CIAT). Colombia. (345): $52 \mathrm{p}$ 
CHOI, W.I., LEE, E.H., CHOI, B.R., PARK, H.M. y AHN, Y.J. 2003. Toxicity of plant essential oils to Trialeurodes vaporariorum (Homoptera: Aleyrodidae). Journal of Economic Entomology. 96:1479 - 1484.

COOK, S.M., KHAN, Z.R. y PICKETT, J.A. 2007. The use of push-pull strategies in integrated pest management. Annual Review Entomology. 52:375 -400 .

DEHGHANI, M., AHMADI, K. y ZOHDI, HADI. 2012. Evaluation of some plant extracts and conventional insecticides against Trialeurodes vaporariorum (westwood) (Homoptera: Aleyrodidae) in greenhouse condition. Munis Entomology \& Zoology. 7(2):828 - 836.

DILLON, P.M., STUART, L. y DOYLE, M. 1983. Mechanical Defenses of Its Host Plant, Cnidoscolus urens (Euphorbiaceae). pp, 112-116. In: Biotropica. Quinceava edición. The Association for Tropical Biology and Conservation Stable. (USA), 359 p.

FEGET, G. 1994. Hidrolatos de Plantas Cultivadas Biológicamente y su Utilización en la Producción de Cultivos. "Una forma innovadora de manejo agrícola. Santafé de Bogotá. 302 p.

FOSTER, S.P. y HARRIS, M.O. 1997. Behavioral manipulation methods for insect pest management. Annual Review Entomology. 42:123 - 146.

FRAGOSO, D.B., GUEDES, M.C. y PICANÇO, L. 2002. Insecticide use and organophosphate resistance in the coffee leaf miner Leucoptera coffeella (Lepidoptera: Lyonetiidae). Bulletin of Entomological Research (UK). 92:203 - 212.

FRASER, H. 2005. Reduced-risk pesticides and Biopesticides. In: Government of Ontario. Eds. OMAFRA. Canada. 28 - 53 p.

GÓMEZ, C. y SOTO, A. 2008. Productos alternativos para el manejo de Cosmopolites sordidus (Coleoptera: Dryophthoridae). Revista Agronomía. 16(2):45 - 50.
HAMDI, F., FARGUES, J., RIDRAY, G., JEANNEQUIN, B. y BONATO, O. 2011. Compatibility among entomopathogenic hyphocreales and two beneficial insects used to control Trialeurodes vaporariorum (Hemiptera: Aleyrodidae) in Mediterranean greenhouses. Journal of Invertebrate Pathology. 108:22 - 29.

ISMAN, B. 2006. Botanical Insecticides, Deterrents and Repellents in Modern Agriculture and an Increasingly Regulated World. Journal of Entomology. 51:45 - 66 .

LANNACONE, J. y LAMAS, G. 2003. Efecto insecticida de cuatro extractos botánicos y del cartap sobre la polilla de la papa Phthorimaea operculella (Zeller) (Lepidoptera: Gelechiidae), en el Perú. Entomotropica. (Venezuela). 18(2):95 - 105.

MOREAU, L. y ISMAN, B. 2012. Trapping whiteflies: A comparison of greenhouse whitefly (Trialeurodes vaporariorum) responses to trap crops and yellow sticky traps. Pest Management Science. 67:408 - 413.

PARK, D., KIM, I. y PARK, U. 1998. Occurrence and within-plant distribution of Trialeurodes vaporariorum (Westwood) and Encarsia formosa (Gahan) in greenhouse. Journal of Applied Entomology. 37:117 $-121$.

PAVELA, R. y HERDA, G. 2007. Repellent effects of pongam oil on settlement and oviposition of the common greenhouse whitefly Trialeurodes vaporariorum on chrysanthemum. Journal of Insect Science. 14:219 - 224.

PÉREZ, E. 2012. Plaguicidas botánicos: Una alternativa a tener en cuenta. Fitosanidad. 16 (1):51 - 59.

PICANÇO, M.C., SOTO, A., BACCI, L., FIDELIS, E.G., SILVA, G.A. y De SENA, M.E. 2007. Controle biológico das principais pragas de hortaliças no Brasil. In: ZAMBOLIM, L. (Ed.). Manejo integrado de doenças e pragas hortaliças. Viçosa: UFV. 505 $537 \mathrm{p}$. 
REGNAULT-ROGER， C., PHILOGÉNE, B. y VINCENT, C. 2005. Biopesticides of Plant Origin. Intercept-Lavoisier. 5:133 - 149.

RODRÍGUEZ, H.C. 2002. Plantas contra plagas. Potencial práctico de ajo, anona, nim, chile y tabaco. Ed. RAAPAN. México. 133p.

ROEL, A.R., VENDRAMIM, J.D., FRIGUETTO, R.T.S. y FRIGUETTO, E. N. 2000. Efeito do extracto acetato de etila de Trichilia pallida Swartz (Meliaceae) no desenvolvimento e sobrevivência da lagarta-docartucho. Bragant. 59:53 - 58.

SAFER. 2014. Agrobiológicos: Producto Alisin. Ficha técnica 01. En: http://www.agrobiologicossafer. com/index.php/productos/extractos-de-plantas/ item/96-alisin.html.html. 1-6pp; consulta: febrero, 2014.

SALAZAR, G.C., BETANCOURTH, C.A. y BACCA. I.T. 2003. Evaluación de extractos vegetales sobre la mosca blanca (Trialeurodes vaporariorum) en frijol en condiciones de laboratorio. Revista de Ciencias Agrícolas. 2(1):51 - 61 .

SCHUSTER, J.,THOMPSON, S., ORTEGA, D. y POLSTON, E. 2009. Laboratory evaluation of products to reduce settling of sweet potato whitefly adults. Journal of Economic Entomology. 102:1482 1489.

SHESKIN, D.J. 2007. Handbook of parametric and non parametric statistical procedures. Ed. $4^{\text {th }}$ Eds. Chapman \& Hall/CRC. USA. 2007.1736p.

SIMMONDS, J., MANLOVE, D., BLANEY, M. y KHAMBAY, S. 2002. Effects of selected botanical insecticides on the behaviour and mortality of the glasshouse. Entomología Experimentalis et Applicata.102: 39 - 47.

SOTO, A., OLIVEIRA, H. y PALLINI, A. 2011. Integración de control biológico y de productos alternativos contra Tetranychus urticae (Acari: Tetranychidae). Revista U.D.C.A. Actualidad y Divulgación Cientifica. 14(1):23 - 29.
VAN LENTEREN, C. y NOLDUS, J. 1990. Whiteflyplant relationships: behavioral and ecological aspects. In: Gerling, D. (Ed.), Whiteflies: their Bionomics, Pest Status and Management. Ed. Intercept Ltd, Hants. (UK). $47-89$ p.

VAN LENTEREN, C., VAN ROERMUND, W. y SÜTTERLIN, S. 1996. Biological control of Greenhouse whitefly (Trialeurodes vaporariorum) with the Parasitoid Encarsia formosa: How does it work?. Biological Control.USA. 6:1 - 10.

WALPOLE, R.E.; MYERS, R.H.; MYERS, S.L. y Ye, K. 2012. Probabilidad y Estadística para Ingeniería y Ciencias. Ed. $9^{\text {th }}$. Eds. Pearson Educación. México. $816 \mathrm{p}$.

ZANDI, N., HOJJATI, M. y CARBONELL, A. 2012. Bioactividad de aceite esencial de Lantana cámara L. contra Callosobruchus maculatus (Fabricius). Revista Chilena de Investigación Agrícola. 72:4 - 6. 